\title{
Organ-Specific Treatment for COVID-19: Rationale, Evidence, and Potential Candidates
}

Organ-Specific Treatment for COVID-19

Mojdeh Rahmanian ${ }^{\text {a }}$, Seyedeh Zahra Mousavi ${ }^{\text {b }}$, Ashkan Sami ${ }^{\text {a }}$ *

${ }^{a}$ Department of Computer Science and Engineering and IT, Shiraz University, Shiraz, Iran

${ }^{b}$ Shiraz University of Medical Sciences, Shiraz, Iran

Corresponding author

*Ashkan Sami. Department of Computer Science and Engineering and IT, Shiraz University, Shiraz, Iran.

Tel: $+98-71-36133569$

Fax: $+98-71-36474605$

Email:sami@shirazu.ac.ir

The ORCID ID: 0000-0002-0023-9543

Word count: 3353 words, excluding abstract, methods, references, table caption, and figure legends.

Data availability statement: The data that support the findings of this study are available from the corresponding author upon reasonable request.

Acknowledgments: This research received no specific grant from any funding agency in the public, commercial, or not-for-profit sectors.

Conflict of interest statement: The authors declare that there are no conflicts of interest. All authors deny any financial and personal relationships with other people or organizations that could inappropriately influence this work.

\section{Author contribution statement:}

- Mojdeh Rahmanian has made substantial contributions to conceptualization and study design, data acquisition and software, and writing the original draft.

- Seyedeh Zahra Mousavi has made substantial contributions to conceptualization and study design, formal analysis and interpretation of data, and writing the original draft.

- Ashkan Sami has made substantial contributions to conceptualization and study design, supervision, and revising \& editing the manuscript.

All authors have approved the final version to be published. 


\begin{abstract}
Background and Purpose: COVID-19 has become the ongoing public health crisis of our time. Although it was first presented as a respiratory infection, extrapulmonary manifestations are increasingly reported. However, no effective therapeutic strategy for COVID-19 extrapulmonary involvement is currently available. The current study aims to analyze the pathogenesis of COVID-19 extrapulmonary complications to evaluate the rationale for proposing organ-specific treatment as a novel therapeutic strategy to manage these multisystemic complications.

Experimental Approach: In this study, differentially expressed genes (DEGs) of SARS-CoV2 infected extrapulmonary organs including human pluripotent stem cells (hPSCs)-derived liver organoids, hPSCs-derived pancreatic endocrine cells, and human-induced pluripotent stem cells (hiPSCs)-derived choroid plexus organoids were analyzed. First, pathway enrichment analysis is done based on the identified DEGs to compare the underlying biological pathways enriched upon SARS-CoV-2 infection in different organs to confirm the need for developing organ-specific treatment strategies. Then, these lists of DEGs are used in a connectivity map-based drug repurposing experiment to propose novel organ-specific therapeutic options.

Key Results: The results reveal different biological pathways and networks responsible for SARS-CoV-2 multisystemic pathogenesis based on the organ involved that highlight the need for considering organ-specific treatments. Besides, some FDA-approved drugs are proposed as the potential therapeutic candidates for each infected cell line.

Conclusion and Implications: Although COVID-19 extrapulmonary manifestations are increasing, management of these complications is still challenging. Traditional therapeutic strategies and already repurposed antiviral agents are not effective. In this situation, organspecific treatment, or in other words personalized therapy might be a promising solution.
\end{abstract}

\title{
What is already known:
}

- Although COVID-19 multiorgan involvement is increasingly reported, no specific treatment is available.

- The mechanism of SARS-CoV-2 induced multiorgan involvement is poorly understood, limiting identifying potential treatments.

\section{What this study adds:}

- This study provides a comparative analysis of the pathogenesis of COVID-19 extrapulmonary infection.

- This study highlights the need for applying organ-specific treatment and proposes potential candidates.

What is the clinical significance:

- Organ-specific treatment would be proposed as a novel therapeutic strategy against the COVID-19 pandemic.

Keywords: COVID-19; SARS-CoV-2; organ-specific treatment; personalized medicine; drug repurposing 


\section{Introduction}

Coronavirus Disease 2019 (COVID-19) is the ongoing global health crisis of our time. It was first reported in December 2019 in China with cases of unknown origin pneumonia with a history of exposure to a seafood market in Wuhan, furtherly spread throughout the world. The pathogen was identified to be a novel member of the betacoronavirus family named Severe Acute Respiratory Syndrome Coronavirus 2 (SARS-CoV-2). On 30 January 2020, the World Health Organization (WHO) declared the COVID-19 outbreak as a global pandemic (Sun, He, et al., 2020; Zhu, Zhang, et al., 2020). As of 6 November 2020, over 48.5 million confirmed COVID-19 cases and 1.2 million deaths have been reported worldwide (WHO, 2020).

COVID-19 patients can present with a wide spectrum of clinical manifestations, most of them are non-specific symptoms. Lovato, de Filippis (2020) conducted a systematic review of available literature on clinical manifestations of COVID-19 patients. A total of 1556 hospitalized COVID-19 cases evaluated in five separate retrospective studies were included in this review. The most common symptoms were fever (85.6\%), cough (68.7\%), fatigue (39.4\%), and shortness of breath $(21.4 \%)$.

These initial studies showed COVID-19 as a respiratory tract infection. However, recent investigations have revealed the multisystemic nature of this newly emerged virus (Zaim, Chong, et al., 2020; Robba, Battaglini, et al., 2020). Gavriatopoulou, Korompoki, et al. (2020) have conducted a review of available literature on COVID-19 multi-organ involvement. They believed that the expression of ACE-2, SARS-CoV-2 receptor, on several human organs, would confirm the multisystemic involvement in COVID-19 patients. Considering already published articles, the most common organ-specific clinical manifestations of COVID-19 can be classified as:

1. Cardiovascular manifestations: COVID-19 patients with pre-existing cardiovascular comorbidities showed a higher risk of developing adverse outcomes (Zhou, Yu, et al., 2020). Besides, COVID-19 patients are at increased risk of developing cardiac complications associated with poor outcomes. Studies have reported a high prevalence of heart failure, cardiogenic shock, arrhythmias, myocarditis, and acute coronary syndrome (ACS) in COVID-19 patients (Shafi, Shaikh, et al., 2020; Samidurai, Das, 2020). Also, some of the proposed COVID-19 therapies have cardiovascular adverse effects, aggravating these complications.

2. Liver and gastrointestinal manifestations: Some COVID-19 patients have presented with gastrointestinal symptoms including anorexia, nausea and vomiting, diarrhea, and abdominal pain in the disease course (Wong, Lui, et al., 2020). Although available data on the prevalence of gastrointestinal symptoms among COVID-19 patients vary significantly, published data from Wuhan revealed that up to $79 \%$ of patients developed gastrointestinal features in the course of the disease (Fang, Guan, et al., 2020). Different theories have been proposed for the mechanism of gastrointestinal involvement in COVID-19 patients including the direct viral attack to epithelial cells, the viral entry with ACE-2 receptor, and mucosal injury due to immune reaction or hypoxemia; however, the exact mechanism is still unknown (Tian, Rong, et al., 2020). Besides, liver dysfunction and abnormal liver function tests were also reported in COVID-19 patients during disease progression (Zhang, Shi, et al., 2020). 
3. Neurologic involvement: There are increasing reports of COVID-19 associated neurological and neuropsychiatric complications. Several neurological syndromes including encephalopathy, ischemic stroke, meningoencephalitis, Guillain-Barre syndrome (GBS), etc. have been reported in COVID-19 patients (Paterson, Brown, et al., 2020; Bridwell, Long, et al., 2020). However, the management of these neurological complications is still challenging.

4. Renal manifestations: Available reports have shown a high prevalence of acute kidney injury (AKI), proteinuria, and hematuria among COVID-19 patients (Chan, Chaudhary, et al., 2020; Kunutsor, Laukkanen, 2020). In a study conducted by Sharma, Uppal, et al. (2020), ten samples of kidney biopsies of COVID-19 patients with the clinical diagnosis of AKI were evaluated. All ten samples showed evidence of acute tubular necrosis (ATN). However, other findings such as pauci-immune crescentic glomerulonephritis (GN), segmental glomerulosclerosis, and thrombotic microangiopathy were also identified.

5. Others: Other less common manifestations such as skin and ophthalmic complications have been reported (Bertoli, Veritti, et al., 2020; Ma, Li, et al., 2020; Zhao, Fang, 2020).

However, ongoing clinical trials have not revealed any effective therapeutic intervention for COVID-19 cases yet. Most of the known antiviral agents already used for other indications did not show significant therapeutic potentials. These challenges would highlight the urgent need to change the treatment strategies and confirm that traditional therapeutic strategies are not effective for this emerging viral disease. However, to propose novel therapeutic strategies, some challenges still exist that should be solved.

First, the mechanism of SARS-CoV-2 multiorgan involvement is still unknown. Although some theories have been proposed for mechanisms of COVID-19 multisystemic manifestations, it is not exactly known whether the virus infects other cell lines with the same mechanism as respiratory tract cells or not (Gupta, Madhavan, et al., 2020). Limited understanding of SARS-CoV-2 multiorgan involvement would justify the ineffectiveness of common antiviral agents.

Besides, although COVID-19 extrapulmonary involvement is increasing, most of the investigational experiments on potential treatments have been still conducted based on data of SARS-CoV-2 infected respiratory tract cell lines. No experiment that analyzes COVID-19 extrapulmonary involvement to identify possible therapeutic interventions exist. Also, patients with multiorgan involvement are receiving the same therapies as cases presenting with typical COVID-19 pneumonia, most of them are based on supportive care (Gupta, Madhavan, et al., 2020). Considering all these challenges, more studies should be conducted to identify mechanisms of SARS-CoV-2 multiorgan involvement and if these studies reveal different mechanisms and pathways, organ-specific treatment should be considered as the novel effective therapeutic strategy against this pandemic.

Besides, the need for applying COVID-19 personalized therapy should also be considered. COVID-19 can present with a wide spectrum of clinical manifestations from asymptomatic cases to severe life-threatening complications. It is not exactly understood why many COVID19 cases have no signs or symptoms, while others are hospitalized or died from the disease. This issue is more prominent among different families. As it is seen recently, several infected members of a family would develop life-threatening complications and die from the disease. 
Therefore, the genes would be the possible key factors to determine the severity and further complications of the disease. As Zhang, Bastard, et al. (2020) showed, inborn genetic mutations of type I interferon (IFN) would be responsible for severe and life-threatening COVID-19 infections. Therefore, analyzing the transcriptome of infected cases to propose potential therapeutic options based on their genomic signatures would be the optimal strategy towards developing COVID-19 personalized therapy. However, considering the genomic similarity of cases of the same family or nationality and limited availability of transcriptome analysis, applying this approach at the family or regional level would be more reasonable.

Since available experiments analyzing infected extrapulmonary tissues are scant, in this study, we aimed to analyze transcriptomic signatures of SARS-CoV-2 infected cell lines belonging to different organs to compare the genes and their associated functional pathways responsible for viral invasion and pathogenesis in each organ to identify whether the virus infects different organs in the same way and if there is a rationale for considering an organspecific treatment. Transcriptomic signature is a list of genes that are significantly up- or downregulated in a specific disease known as differentially expressed genes (DEGs) reflecting the biologic mechanisms involved in disease pathogenesis.

Besides, we set out to perform a drug repurposing experiment to propose COVID-19 organspecific therapeutic options. Transcriptomic signatures of SARS-CoV-2 infected cells were compared with genomic signatures of cell lines treated with various compounds known as connectivity map (CMap) (Qu, Rajpal, 2012). CMap libraries include gene expression profiles of human cell lines perturbed with various compounds in comparison to their mock-treated samples. Results of the CMap analysis would be shown with a connectivity-score for each drug-disease pair ranging from -1 to +1 . A negative connectivity-score shows that the compound can reverse the genomic signature of the disease; so, the compound can be proposed as the therapeutic option for the mentioned disease (Musa, Ghoraie, et al., 2018).

\section{Materials and methods}

\section{Dataset selection}

Gene Expression Omnibus (GEO) database of National Center for Biotechnology Information (NCBI) (http://www.ncbi.nlm.nih.gov/geo/) (Barrett, Troup, et al., 2007), the largest public genomics data repository, was searched for "COVID-19" or "SARS-CoV-2" related datasets. 1575 datasets containing these terms were found that 1335 of them were conducted on "homo sapiens" and selected for further analysis. These selected datasets were manually searched for datasets that contained transcriptomic data of SARS-CoV-2 infected extrapulmonary organs. Five datasets were identified (i.e. GSE156754, GSE150392, GSE149312, GSE151803, and GSE157852).

Since the purpose of this study is to analyze and compare SARS-CoV-2 pathogenesis in different human organs and no dataset that contains the transcriptomic analysis of different organs exists, more than one dataset should be selected. However, the overall design of these 
selected datasets including cell growth protocols, extraction protocols, and data processing should be as similar as possible to validate the results. Therefore, GSE151803 and GSE157852 were selected since both datasets have analyzed the expression profiling of mock-infected versus SARS-CoV-2 infected homo sapiens stem cell-derived cells/organoids by high throughput sequencing at the same time points.

Briefly, the GSE151803 (Yang, Han, et al., 2020) contains the transcriptomic analysis of SARS-CoV-2 infected versus mock-treated human pluripotent stem cells (hPSCs)-derived cells and organoids including pancreatic endocrine cells and liver organoids 24 hours postinfection (hpi). The GSE157852 (Jacob, Pather, et al., 2020) includes transcriptomic analysis of human-induced pluripotent stem cells (hiPSCs)-derived choroid plexus organoids that were mock-treated or infected with SARS-CoV-2, at 24 and $72 \mathrm{hpi}$. Since these two selected datasets should have the same protocols, we only focused on data collected at $24 \mathrm{hpi}$. For the differential expression analysis of these datasets, genes with $\mathrm{p}$-value $<0.05$ and |fold-changel $>2$ were identified as DEGs.

Since neurologic and gastrointestinal complications are common manifestations of COVID19 multiorgan involvement, these two datasets would enhance our understanding of this virus's multisystemic nature.

\section{Gene analysis and Functional pathway enrichment}

The identified DEGs were analyzed with the Metascape web tool (http://metascape.org/). Metascape is an online gene annotation and analysis tool designed to provide biological interpretations of OMICs data (Zhou. Y, Zhou. B, et al., 2019). Metascape workflow contains gene annotation, membership, functional enrichment, and interactome analysis. Lists of DEGs resulting from SARS-CoV-2 infected vs mock-treated hPSCs-derived liver organoids, hPSCsderived pancreatic endocrine cells, and hiPSCs-derived choroid plexus organoids were uploaded in Metascape web tool to perform the multi-gene-list meta-analysis. Metascape can perform comparative approaches to compare these three gene lists and show overlaps and discrepancies of these OMICs data even at biologic pathways and protein levels.

First, the up-regulated genes of these three cell lines were uploaded separately. Gene lists were compared to identify the overlaps at the gene level. Then, pathway enrichment analysis was performed for each given gene list with the following ontology sources: KEGG Pathway, GO Biological Processes, Reactome Gene Sets, Canonical Pathways, and CORUM. Terms with a p-value $<0.01$, a minimum count of 3 , and an enrichment factor $>1.5$ were grouped into clusters based on hierarchical clustering. Kappa scores were used as the similarity metric. Subtrees with a similarity score of $>0.3$ were considered a cluster. The most statistically significant term within each cluster was chosen to represent the cluster. Enrichment factor is the ratio between the number of the given pathway members found in our gene list in comparison to counts observed by chance. The p-value is calculated based on the cumulative hypergeometric distribution and means the probability of obtaining the observed or more pathway members in our gene list. Since no approved cut-offs for these metrics exist and thresholds of these metrics such as enrichment factor, p-value, kappa score, etc. depend on the purpose of each study and distribution of the results (Reimand, Isserlin, et al., 2019), Metascape has defined these default 
values for these metrics cut-offs based on previous statistical analysis and many papers have recently used these metrics with these cut-offs through the Metascape web tool with reliable and valid results (Battaglia, Venturin, et al., 2019; Wang, Shi, et al., 2019).

The pathways enriched in each gene list were furtherly compared. This comparison would reveal whether the underlying biological pathways and mechanisms associated with COVID19 infection are the same in different organs or the virus infects different organs through different mechanisms. All the mentioned steps were furtherly repeated with the down-regulated genes.

\section{CMap analysis}

The "CMap build 02" (https://portals.broadinstitute.org/cmap/), available in the PharmacoGx package, was used for connectivity map analysis. PharmacoGx is an open-source $\mathrm{R}$ package for integrative analysis of large-scale pharmacogenomics data (Smirnov, Safikhani, et al., 2016). "CMap build 02" contains 7,056 gene expression profiles of five different human cell lines treated with 1,309 compounds, most of which are the United States Food and Drug Administration (FDA)-approved drugs (Montero-Melendez, Perretti, 2014).

Lists of the identified DEGs of SARS-CoV-2 infected hPSCs-derived liver organoids, hPSCs-derived pancreatic endocrine cells, and hiPSCs-derived choroid plexus organoids were uploaded as the input data separately followed by querying the CMap library of gene expression profiles. Each uploaded gene list was compared with the gene expression signatures of different perturbations or compounds by a pattern-matching algorithm based on the nonparametric rank-ordered Kolmogorov-Smirnov (Hollander, Wolfe, et al., 2013; Cheng, Yang, et al., 2014). The results were presented with a connectivity score for each drug-disease pair, ranging from -1 to +1 . The more negative score revealed the greater potential of that compound to reverse the genomic signature of the disease; therefore, the compound can be proposed as the potential treatment for that specific disease.

\section{Results}

\section{Dataset selection}

We aimed to analyze the genes differentially expressed upon SARS-CoV-2 infection, in hPSCs-derived pancreatic endocrine cells, hPSCs-derived liver organoids, and hiPSCs-derived choroid plexus organoids 24 hpi available in GSE151803 and GSE157852. All genes with pvalue $<0.05$ and |fold-changel $>2$ were selected as DEGs.

A total of 145 genes were identified to be modulated by SARS-CoV-2 infection in hPSCsderived pancreatic endocrine cells (68 up-regulated and 77 down-regulated genes). For hPSCsderived liver organoids, a total of 1039 genes were modulated by SARS-CoV-2 infection (499 up-regulated and 540 down-regulated genes) and for hiPSCs-derived choroid plexus organoids, a total of 1256 genes were differentially expressed upon SARS-CoV-2 infection (317 upregulated and 939 down-regulated genes). These lists of DEGs were furtherly analyzed through

pathway enrichment and CMap analysis. 


\section{Gene analysis and Functional pathway enrichment}

The identified lists of DEGs were analyzed with the Metascape web tool. First, gene analysis and pathway enrichment were done based on up-regulated genes. Figure 1 showed the overlaps between the up-regulated genes of the three selected cell lines with a Circos plot. As the figure showed, only one gene was included in all three lists. $90 \%$ of up-regulated genes of SARSCoV-2 infected hPSCs-derived liver organoids and hiPSCs-derived choroid plexus organoids and $72 \%$ of up-regulated genes of SARS-CoV-2 infected hPSCs-derived pancreatic endocrine cells were unique to their associated cells.

Then, biological pathway enrichment analysis was carried out for up-regulated genes of each cell line separately and the top enriched terms were compared. Figure 2 showed the heatmap of enriched terms across the three input gene lists. Up-regulated genes in SARS-CoV2 infected hPSCs-derived liver organoids were mainly enriched in "response to molecule of bacterial origin" and "response to interferon-gamma". However, the most significantly enriched pathways in up-regulated genes of SARS-CoV-2 infected hiPSCs-derived choroid plexus organoids were "response to wounding", "positive regulation of cell migration", "blood vessel development", and "extracellular matrix organization". Furthermore, the "pantothenate metabolic process" was the most enriched term in up-regulated genes of infected hPSCsderived pancreatic endocrine cells.

Then, down-regulated genes were uploaded. As figure 3 showed, down-regulated genes of infected hPSCs-derived pancreatic endocrine cells and hiPSCs-derived choroid plexus organoids do not have any overlap at the gene level. Results of pathway enrichment analysis of down-regulated genes of these three cell lines were shown in figure 4. For infected hPSCsderived liver organoids, "monocarboxylic acid metabolic process", "organic hydroxy compound metabolic process", and "biological oxidations" were the most enriched biologic terms. Down-regulated genes of infected hiPSCs-derived choroid plexus organoids were mainly enriched in "cilium organization", "generation of precursor metabolites and energy", and "monocarboxylic acid metabolic process". However, down-regulated genes of infected hPSCs-derived pancreatic endocrine cells were enriched in "spliceosomal tri-snRNP complex assembly".

\section{CMap analysis}

CMap analysis was conducted with the "CMap build 02" library of the PharmacoGx package. Compounds with $\mathrm{p}$-value $<0.05$ and negative connectivity scores were selected as potential therapeutic options.

Nine compounds were identified to significantly reverse transcriptomic signatures of SARS-CoV-2 infected hPSCs-derived pancreatic endocrine cells; "quizapine", "delsoline", and "l-methionine sulfoximine" had the most negative connectivity-scores. Seven compounds including "fasudil", "halcinonide", "flurbiprofen", "midodrine", "mevalolactone", "mepenzolate bromide", and "bephenium hydroxyl naphthoate" significantly reverse genomic signatures of infected hPSCs-derived liver organoids and can be selected for further in vitro and in vivo studies. Besides, 60 FDA-approved drugs were identified as potential therapeutic agents for SARS-CoV-2 infected hiPSCs-derived choroid plexus organoids based on the CMap 
results that "iloprost", "hydroxyachillin", "alpha-ergocryptine", and "lansoprazole" had the highest scores. Seven resulting top-scoring compounds for each organ have been summarized in table 1.

\section{Discussion}

As of 6 November 2020, SARS-CoV-2 has infected more than 48.5 million people from over 235 countries. Although it was initially presented as respiratory disease, many patients have developed extrapulmonary manifestations.

COVID-19 patients can present with cardiovascular manifestations and in particular cases with pre-existing cardiac comorbidities are at increased risk. However, the underlying pathophysiology is not exactly known. While isolation of the virus from autopsy samples of myocardial tissues was in favor of direct virus-induced damage (Tavazzi, Pellegrini, et al., 2020), some other studies have proposed the role of systemic inflammation and endothelial damage in cardiovascular complications (Sala, Peretto, et al., 2020; Xu, Shi, et al., 2020).

Gastrointestinal and hepatobiliary sequels including elevated levels of aminotransferases and bilirubin were also reported (Zhou, Yu, et al., 2020; Guan, Ni, et al., 2020; Bhatraju, Ghassemieh, et al., 2020). Neurologic complications are increasingly observed. A wide spectrum of neurologic presentations including headache, dizziness, anosmia, ageusia, and more life-threatening complications such as stroke, acute hemorrhagic necrotizing encephalitis, encephalopathy, and Guillain-Barre syndrome has been reported (Paterson, Brown, et al., 2020; Bridwell, Long, et al., 2020; Aghagoli, Marin, et al., 2020).

However, key mechanisms responsible for COVID-19 extra-pulmonary involvement are not fully understood. Direct viral invasion, immune dysregulation, endothelial damage, and dysregulation of the Renin-Angiotensin-Aldosterone System (RAAS) can play significant roles in COVID-19 multiorgan damage (Gupta, Madhavan, et al., 2020). Although the management of cases with extrapulmonary manifestations is mainly based on supportive care, poor understanding of the pathophysiology of SARS-CoV-2 induced multiorgan complications has limited the development of prophylactic or therapeutic interventions.

As Gupta, Madhavan, et al. (2020) summarized, more studies are urgently needed to answer some critical questions. We do not exactly know what is the pathophysiology of COVID-19 multiorgan damage and whether the virus infects extrapulmonary organs with the same predominant mechanisms as pulmonary tissues or not. However, studies conducted to solve these poorly-understood challenges are limited.

To answer some of these challenges, we analyzed the transcriptomic signature of SARSCoV-2 infected extrapulmonary organs. Since the pathogen would change the genomic expression of the infected cells in the way optimal for its proliferation, performing analysis at the gene level would be a reasonable approach to understand pathways underlying the infection. Transcriptomic analysis or genome-wide gene expression profiling can identify DEGs and associated networks involved in disease pathogenesis, discover novel targets for diagnostic and therapeutic interventions, and identify significantly up- or down-regulated genes in response to a perturbation such as a chemical compound.

Comparing transcriptomic profiling of SARS-CoV-2 infected extrapulmonary organs in this study has revealed that functional biological pathways enriched during the infection and the 
host responses vary substantially among different organs. This evidence strongly supports the theory that the virus involves various organs with different underlying mechanisms and pathophysiology. Therefore, these results revealed that applying the same treatments for patients with the involvement of different organs is not reasonable and strongly highlighted the need for organ-specific treatments. However, datasets used in this study have analyzed the transcriptomic signature 24 hours post-infection. Further studies should include data obtained at different time points post-infection to evaluate late-phase host responses and dynamics of viral pathogenesis.

Besides, we conducted a CMap-based drug repurposing approach. The CMap compares the gene expression signature of a phenotype of interest such as a disease with a large catalog of gene expression signatures of cells treated with different compounds to find drug-disease connections. Applying this approach in this study has identified novel options for COVID-19 organ-specific treatment, most of them can be justified with available literature. This study is the first experiment that has confirmed the need for organ-specific treatment and identified potential therapeutic options for each infected organ.

"Fasudil", a Rho kinase inhibitor, showed the highest connectivity-score to reverse the genomic signature of SARS-CoV-2 infected hPSCs-derived liver organoids. Abedi, Hayes, et al. (2020) have reviewed the role of Rho kinase in acute lung injury (ALI) and acute respiratory distress syndrome (ARDS). They mentioned that up-regulation of Rho kinase signaling pathways would contribute to immune cell migration, apoptosis, coagulation, contraction, cell adhesion in pulmonary endothelial cells, and finally result in endothelium dysfunction and pulmonary edema. Therefore, Rho kinase inhibitors such as fasudil can reverse these pathways. Considering the pathophysiology of COVID-19 pulmonary damage, fasudil was proposed as the potential prophylactic and therapeutic agent for critically ill patients (Abedi, Rezaee, et al., 2020).

Besides, Bale, Doneen, et al. (2020) hypothesized the role of microvascular disease in COVID-19 complications. They believed that Rho kinase inhibitors such as fasudil can be beneficial for COVID-19 patients with evidence of microvascular involvement since it was found that Rho kinase inhibitors can be used as the therapeutic options for patients with microvascular angina (Berry, Sidik, et al., 2019).

Interestingly, previous experiments have shown substantial effectiveness of fasudil for a wide range of liver injuries. In a study conducted by Thorlacius, Slotta, et al. (2006), fasudil was proposed as a potential therapeutic agent for septic liver injury. They found that treatment with fasudil reduces leukocyte adhesion, chemokine production in the liver of infected mice, and hepatocellular apoptosis besides increasing sinusoidal perfusion in mice models. More recent studies have revealed the protective effect of fasudil from ischemia/reperfusion injury on hepatocytes and it's potential to reduce hepatocyte fibrosis in rat models (Xie, Zhao, et al., 2018; Zhou, Fang, et al., 2014). All these mentioned studies would confirm the effectiveness of fasudil administration for patients with signs of liver involvement.

"Iloprost", a synthetic analog of prostacyclin PGI2, was resulted as a potential therapeutic option for SARS-CoV-2 infected hiPSCs-derived choroid plexus organoids. Autopsy samples of COVID-19 patients have shown evidence of endotheliitis in pulmonary capillaries (Ackermann, Verleden, et al., 2020). Endotheliitis would result in vasoconstriction, endothelial dysfunction, and a hypercoagulable state (Varga, Flammer, et al., 2020). Since iloprost 
possesses antithrombotic, anti-inflammatory, and anti-fibrotic properties, it was proposed as the adjunctive therapy for COVID-19-associated vasculopathy (Stratton, Shiwen, et al., 2001; Czeslick, Simm, et al., 2003). Moezinia, Ji-Xu, et al. (2020) have added iloprost to standard care of three COVID-19 hospitalized patients. They revealed that iloprost can improve digital ischemia and cardiorespiratory parameters in patients with COVID-19-related vasculopathy.

Besides, the effect of iloprost on the brain was under investigation for a few decades. Palaoglu, Erbengi, et al. (1990) have revealed the cytoprotective effect of iloprost on brain tissue grafts of rats in 1990. In another study conducted in 2006 (Aytac, Seymen, et al., 2006), cases of bilateral carotid artery occlusion were treated with iloprost. The results suggested the protective effect of iloprost on the brain after the oxidative stress following cerebral hypoperfusion. More studies have confirmed the protective role of this compound on brain tissue (Mathieu, Sanna, et al., 2002; Dog̃an, Temiz, et al., 1996). This evidence would support the effectiveness of iloprost for brain tissue damage.

We have previously conducted a CMap-based drug repurposing study to identify potential therapeutic candidates for SARS-CoV-2 infected primary human lung epithelial cell line (NHBE) and transformed lung alveolar cell line (A549) (Mousavi, Rahmanian, et al., 2020). Interestingly, some of the compounds that resulted in this current study such as alphaergocryptine, lansoprazole, halcinonide, and midodrine were also found as potential treatments for infected A549 and NHBE cell lines. However, most of the resulting compounds differ substantially based on the organ involved. These results can reveal that although the pathways underlying SARS-CoV-2 pathogenesis in different organs share some similarities, the virus uses different mechanisms to infect the cells based on the organ involved.

Furthermore, since this study revealed different genes and biologic pathways responsible for COVID-19 multiorgan involvement, proposing novel methods to apply personalized therapy should also be considered. Patients from different nationalities with various preexisting comorbidities would present with variable symptoms and the genes or the "omics" are the main causes of this variation. The optimal strategy would be to use precision medicine to apply COVID-19 personalized therapy according to the patient's genes. The first step towards this purpose would include comparing the transcriptome of asymptomatic infected patients with cases presenting with more severe diseases that are hospitalized or need invasive mechanical ventilation to propose lead drug candidates based on the severity. Then, the study can be extended to analyze the genomic profiles of cases of the same nationality and infected members of a family with significant genetic similarity to propose the best therapeutic intervention for each patient based on the "omics" data.

\section{Conclusion}

Although COVID-19 extrapulmonary manifestations are increasingly reported, available literature to analyze the underlying mechanisms and propose therapeutic strategies are scant. Transcriptomic analysis of infected organs would reveal the key mechanisms playing role in SARS-CoV-2 pathogenesis. In this study, analyzing genomic signatures of SARS-CoV-2 infected extrapulmonary organs has revealed different underlying pathways and networks responsible for disease pathogenesis based on the organ involved. These results strongly 
highlight the need for applying organ-specific treatments. Besides, conducting a CMap-based drug repurposing study has identified potential therapeutic options for each organ that need further studies to confirm.

\section{References:}

Abedi, F., Hayes, A. W., Reiter, R., \& Karimi, G. (2020). Acute Lung Injury: the therapeutic role of Rho kinase inhibitors. Pharmacological Research, 155, 104736.

Abedi, F., Rezaee, R., \& Karimi, G. (2020). Plausibility of therapeutic effects of Rho kinase inhibitors against Severe Acute Respiratory Syndrome Coronavirus 2 (COVID-19). Pharmacological Research, 156, 104808.

Ackermann, M., Verleden, S. E., Kuehnel, M., Haverich, A., Welte, T., Laenger, F., . . . Tzankov, A. (2020). Pulmonary vascular endothelialitis, thrombosis, and angiogenesis in Covid-19. New England Journal of Medicine, 383, 120-128.

Aghagoli, G., Marin, B. G., Katchur, N. J., Chaves-Sell, F., Asaad, W. F., \& Murphy, S. A. (2020). Neurological involvement in COVID-19 and potential mechanisms: a review. Neurocritical care, 1-10.

Aytac, E., Seymen, H. O., Uzun, H., Dikmen, G., \& Altug, T. (2006). Effects of iloprost on visual evoked potentials and brain tissue oxidative stress after bilateral common carotid artery occlusion. Prostaglandins, leukotrienes and essential fatty acids, 74(6), 373-378.

Bale, B. F., Doneen, A. L., \& Vigerust, D. J. (2020). Microvascular disease confers additional risk to COVID-19 infection. Medical hypotheses, 144, 109999.

Barrett, T., Troup, D. B., Wilhite, S. E., Ledoux, P., Rudnev, D., Evangelista, C., . . Edgar, R. (2007). NCBI GEO: mining tens of millions of expression profiles-database and tools update. Nucleic acids research, 35(suppl_1), D760-D765.

Battaglia, C., Venturin, M., Sojic, A., Jesuthasan, N., Orro, A., Spinelli, R., ... \& Adorni, F. (2019). Candidate genes and MiRNAs linked to the inverse relationship between cancer and Alzheimer's disease: insights from data mining and enrichment analysis. Frontiers in Genetics, 10, 846.

Berry, C., Sidik, N., Pereira, A. C., Ford, T. J., Touyz, R. M., Kaski, J. C., \& Hainsworth, A. H. (2019). Small-vessel disease in the heart and brain: current knowledge, unmet therapeutic need, and future directions. Journal of the American Heart Association, 8(3), e011104.

Bertoli, F., Veritti, D., Danese, C., Samassa, F., Sarao, V., Rassu, N., . . Lanzetta, P. (2020). Ocular Findings in COVID-19 Patients: A Review of Direct Manifestations and Indirect Effects on the Eye. Journal of ophthalmology, 2020, 4827304.

Bhatraju, P. K., Ghassemieh, B. J., Nichols, M., Kim, R., Jerome, K. R., Nalla, A. K., . . . Evans, L. (2020). Covid-19 in critically ill patients in the Seattle region-case series. New England journal of medicine, 382(21), 2012-2022.

Bridwell, R., Long, B., \& Gottlieb, M. (2020). Neurologic complications of COVID-19. The American Journal of Emergency Medicine, 38(7), 1549.e3-1549.e7.

Chan, L., Chaudhary, K., Saha, A., Chauhan, K., Vaid, A., Zhao, S., . . Miotto, R. (2020). AKI in Hospitalized Patients with COVID-19. Journal of the American Society of Nephrology, 31(11), 1032.

Cheng, J., Yang, L., Kumar, V., \& Agarwal, P. (2014). Systematic evaluation of connectivity map for disease indications. Genome medicine, 6(12), 95.

Czeslick, E., Simm, A., Grond, S., Silber, R. E., \& Sablotzki, A. (2003). Inhibition of intracellular tumour necrosis factor (TNF)- $\alpha$ and interleukin (IL)-6 production in 
human monocytes by iloprost. European journal of clinical investigation, 33(11), 10131017.

Dog̃an, A., Temiz, C., Türker, R. K., Egemen, N., \& Başkaya, M. K. (1996). Effect of the prostacyclin analogue, iloprost, on infarct size after permanent focal cerebral ischemia. General Pharmacology: The Vascular System, 27(7), 1163-1166.

Fang, D., MA, J., Guan, J., Wang, M., Song, Y., Tian, D., \& LI, P. (2020). Manifestations of Digestive system in hospitalized patients with novel coronavirus pneumonia in Wuhan, China: a single-center, descriptive study. Chinese Journal of Digestion, 12, E005.

Gavriatopoulou, M., Korompoki, E., Fotiou, D., Ntanasis-Stathopoulos, I., Psaltopoulou, T., Kastritis, E., . . . Dimopoulos, M. A. (2020). Organ-specific manifestations of COVID19 infection. Clinical and experimental medicine, 20, 493-506.

Guan, W.-j., Ni, Z.-y., Hu, Y., Liang, W.-h., Ou, C.-q., He, J.-X., . . Hui, D. S. (2020). Clinical characteristics of coronavirus disease 2019 in China. New England journal of medicine, 382(18), 1708-1720.

Gupta, A., Madhavan, M. V., Sehgal, K., Nair, N., Mahajan, S., Sehrawat, T. S., . . Wan, E. Y. (2020). Extrapulmonary manifestations of COVID-19. Nature medicine, 26(7), 1017-1032.

Hollander, M., Wolfe, D. A., \& Chicken, E. (2013). Nonparametric statistical methods (Vol. 751): John Wiley \& Sons.

Jacob, F., Pather, S. R., Huang, W.-K., Zhang, F., Wong, S. Z. H., Zhou, H., . . Xu, M. (2020). Human pluripotent stem cell-derived neural cells and brain organoids reveal SARSCoV-2 neurotropism predominates in choroid plexus epithelium. Cell stem cell.

Kunutsor, S. K., \& Laukkanen, J. A. (2020). Renal complications in COVID-19: a systematic review and meta-analysis. Annals of Medicine, 52(7), 345-353.

Lovato, A., \& de Filippis, C. (2020). Clinical presentation of COVID-19: a systematic review focusing on upper airway symptoms. Ear, Nose \& Throat Journal, 99(9), 569-576.

Ma, N., Li, P., Wang, X., Yu, Y., Tan, X., Chen, P., . . Jiang, F. (2020). Ocular manifestations and clinical characteristics of children with laboratory-confirmed COVID-19 in Wuhan, China. JAMA ophthalmology, 138(10), 1079-1086.

Mathieu, A., Sanna, G., Mameli, A., Pinna, C., Vacca, A., Cauli, A., . . Piga, M. (2002). Sustained normalization of cerebral blood-flow after iloprost therapy in a patient with neuropsychiatric systemic lupus erythematosus. Lupus, 11(1), 52-56.

Moezinia, C. J., Ji-Xu, A., Azari, A., Horlick, S., Denton, C., \& Stratton, R. (2020). Iloprost for COVID-19-related vasculopathy. The Lancet Rheumatology, 2(10), e582-e583.

Montero-Melendez, T., \& Perretti, M. (2014). Connections in pharmacology: innovation serving translational medicine. Drug discovery today, 19(7), 820-823.

Mousavi, S. Z., Rahmanian, M., \& Sami, A. (2020). A connectivity map-based drug repurposing study and integrative analysis of transcriptomic profiling of SARS-CoV-2 infection. Infection, Genetics and Evolution, 86, 104610.

Musa, A., Ghoraie, L. S., Zhang, S.-D., Glazko, G., Yli-Harja, O., Dehmer, M., . . . EmmertStreib, F. (2018). A review of connectivity map and computational approaches in pharmacogenomics. Briefings in bioinformatics, 19(3), 506-523.

Palaoglu, S., Erbengi, A., Sav, A., Erbengi, T., \& Turker, R. (1990). Cytoprotective effect of iloprost on isolated cortical brain tissue grafts in rats. Prostaglandins, leukotrienes and essential fatty acids, 39(3), 203-206.

Paterson, R. W., Brown, R. L., Benjamin, L., Nortley, R., Wiethoff, S., Bharucha, T., . . . Zambreanu, L. (2020). The emerging spectrum of COVID-19 neurology: clinical, radiological and laboratory findings. Brain, 143(10), 3104-3120.

Qu, X. A., \& Rajpal, D. K. (2012). Applications of Connectivity Map in drug discovery and development. Drug discovery today, 17(23-24), 1289-1298. 
Reimand, J., Isserlin, R., Voisin, V., Kucera, M., Tannus-Lopes, C., Rostamianfar, A., ... \& Merico, D. (2019). Pathway enrichment analysis and visualization of omics data using g: Profiler, GSEA, Cytoscape and EnrichmentMap. Nature protocols, 14(2), 482-517.

Robba, C., Battaglini, D., Pelosi, P., \& Rocco, P. R. (2020). Multiple organ dysfunction in SARS-CoV-2: MODS-CoV-2. Expert review of respiratory medicine, 14(9), 865-868.

Sala, S., Peretto, G., Gramegna, M., Palmisano, A., Villatore, A., Vignale, D., . . . Basso, C. (2020). Acute myocarditis presenting as a reverse Tako-Tsubo syndrome in a patient with SARS-CoV-2 respiratory infection. European Heart Journal, 41(19), 1861-1862.

Samidurai, A., \& Das, A. (2020). Cardiovascular Complications Associated with COVID-19 and Potential Therapeutic Strategies. International journal of molecular sciences, 21(18), 6790.

Shafi, A. M., Shaikh, S. A., Shirke, M. M., Iddawela, S., \& Harky, A. (2020). Cardiac manifestations in COVID-19 patients-A systematic review. Journal of cardiac surgery, 35(8), 1988-2008.

Sharma, P., Uppal, N. N., Wanchoo, R., Shah, H. H., Yang, Y., Parikh, R., . . Jhaveri, K. D. (2020). COVID-19-associated kidney injury: a case series of kidney biopsy findings. Journal of the American Society of Nephrology, 31(9), 1948-1958.

Smirnov, P., Safikhani, Z., El-Hachem, N., Wang, D., She, A., Olsen, C., . . . Grossmann, P. (2016). PharmacoGx: an R package for analysis of large pharmacogenomic datasets. Bioinformatics, 32(8), 1244-1246.

Stratton, R., Shiwen, X., Martini, G., Holmes, A., Leask, A., Haberberger, T., . . Abraham, D. (2001). Iloprost suppresses connective tissue growth factor production in fibroblasts and in the skin of scleroderma patients. The Journal of clinical investigation, 108(2), 241-250.

Sun, J., He, W.-T., Wang, L., Lai, A., Ji, X., Zhai, X., . . Z Zhou, J. (2020). COVID-19: epidemiology, evolution, and cross-disciplinary perspectives. Trends in Molecular Medicine, 26(5), 483-495.

Tavazzi, G., Pellegrini, C., Maurelli, M., Belliato, M., Sciutti, F., Bottazzi, A., . . Bruno, R. (2020). Myocardial localization of coronavirus in COVID-19 cardiogenic shock. European journal of heart failure, 22(5), 911-915.

Thorlacius, K., Slotta, J. E., Laschke, M. W., Wang, Y., Menger, M. D., Jeppsson, B., \& Thorlacius, H. (2006). Protective effect of fasudil, a Rho-kinase inhibitor, on chemokine expression, leukocyte recruitment, and hepatocellular apoptosis in septic liver injury. Journal of leukocyte biology, 79(5), 923-931.

Tian, Y., Rong, L., Nian, W., \& He, Y. (2020). gastrointestinal features in COVID-19 and the possibility of faecal transmission. Alimentary pharmacology \& therapeutics, 51(9), 843-851.

Varga, Z., Flammer, A. J., Steiger, P., Haberecker, M., Andermatt, R., Zinkernagel, A. S., . . . Moch, H. (2020). Endothelial cell infection and endotheliitis in COVID-19. The Lancet, 395(10234), 1417-1418.

Wang, L., Shi, J., Huang, Y., Liu, S., Zhang, J., Ding, H., ... \& Chen, Z. (2019). A six-gene prognostic model predicts overall survival in bladder cancer patients. Cancer cell international, 19(1), 229.

WHO. (2020). WHO Coronavirus Disease (COVID-19) Dashboard. Retrieved from https://covid19.who.int/

Wong, S. H., Lui, R. N., \& Sung, J. J. (2020). Covid-19 and the digestive system. Journal of gastroenterology and hepatology, 35(5), 744-748.

Xie, Y., Zhao, D., Dong, P., \& Lai, L. (2018). Macrophage-targeting Fasudil treatment protects liver from the ischemia/reperfusion injury by promoting M2 macrophage polarization. Bioscience reports. 
Xu, Z., Shi, L., Wang, Y., Zhang, J., Huang, L., Zhang, C., . . . Zhu, L. (2020). Pathological findings of COVID-19 associated with acute respiratory distress syndrome. The Lancet respiratory medicine, 8(4), 420-422.

Yang, L., Han, Y., Nilsson-Payant, B. E., Gupta, V., Wang, P., Duan, X., . . Jaffré, F. (2020). A human pluripotent stem cell-based platform to study SARS-CoV-2 tropism and model virus infection in human cells and organoids. Cell stem cell, 27(1), 125-136. e127.

Zaim, S., Chong, J. H., Sankaranarayanan, V., \& Harky, A. (2020). COVID-19 and multi-organ response. Current Problems in Cardiology, 45(8), 100618.

Zhang, C., Shi, L., \& Wang, F.-S. (2020). Liver injury in COVID-19: management and challenges. The lancet Gastroenterology \& hepatology, 5(5), 428-430.

Zhang, Q., Bastard, P., Liu, Z., Le Pen, J., Moncada-Velez, M., Chen, J., ... \& Rosain, J. (2020). Inborn errors of type I IFN immunity in patients with life-threatening COVID19. Science, 370(6515).

Zhao, Q., Fang, X., Pang, Z., Zhang, B., Liu, H., \& Zhang, F. (2020). COVID-19 and cutaneous manifestations: a systematic review. Journal of the European Academy of Dermatology and Venereology.

Zhou, F., Yu, T., Du, R., Fan, G., Liu, Y., Liu, Z., . . . Gu, X. (2020). Clinical course and risk factors for mortality of adult inpatients with COVID-19 in Wuhan, China: a retrospective cohort study. The lancet, 395(10229), 1054-1062.

Zhou, H., Fang, C., Zhang, L., Deng, Y., Wang, M., \& Meng, F. (2014). Fasudil hydrochloride hydrate, a Rho-kinase inhibitor, ameliorates hepatic fibrosis in rats with type 2 diabetes. Chinese Medical Journal, 127(2), 225-231.

Zhou, Y., Zhou, B., Pache, L., Chang, M., Khodabakhshi, A. H., Tanaseichuk, O., . . Chanda, S. K. (2019). Metascape provides a biologist-oriented resource for the analysis of systems-level datasets. Nature communications, 10(1), 1-10.

Zhu, N., Zhang, D., Wang, W., Li, X., Yang, B., Song, J., . . . Lu, R. (2020). A novel coronavirus from patients with pneumonia in China, 2019. New England Journal of Medicine, 382(8), 727-733. 


\section{Tables and Figures:}

\section{Table 1}

Top-scoring CMap analysis results based on the organ involved. Compounds are ranked based on connectivity-score, ranging from -1 (indicating the completely reverse correlation of signatures between the compound and the disease) to +1 . Resulting investigational molecules without FDA approval have been omitted from this table.

\begin{tabular}{|c|c|c|c|c|c|c|c|c|c|}
\hline & Pancreatic & locrine cells & & Liver organoi & & & Choroid plexus cel & & \\
\hline Rank & Compound & $\begin{array}{l}\text { Connectivity } \\
\text {-score }\end{array}$ & $\begin{array}{l}\text { p- } \\
\text { value }\end{array}$ & Compound & $\begin{array}{l}\text { Connectivity } \\
\text {-score }\end{array}$ & $\begin{array}{l}\text { p- } \\
\text { value }\end{array}$ & Compound & $\begin{array}{l}\text { Connectivity } \\
\text {-score }\end{array}$ & $\begin{array}{l}\text { p- } \\
\text { value }\end{array}$ \\
\hline 1 & quizapine & -0.48473 & 0.031 & fasudil & -0.688825 & 0.021 & iloprost & -0.32592 & 0.013 \\
\hline 2 & delsoline & -0.474905 & 0.021 & halcinonide & -0.244625 & 0.029 & hydroxyachillin & -0.295875 & 0.018 \\
\hline 3 & $\begin{array}{l}\text { L- } \\
\text { methionine } \\
\text { sulfoximine }\end{array}$ & -0.470375 & 0.037 & flurbiprofen & -0.23689 & 0.027 & alpha-ergocryptine & -0.290245 & 0.003 \\
\hline 4 & etofylline & -0.455975 & 0.008 & midodrine & -0.23662 & 0.025 & lansoprazole & -0.283655 & 0.013 \\
\hline 5 & oxprenolol & -0.452075 & 0.035 & mevalolactone & -0.230525 & 0.042 & citalopram & -0.28132 & 0.008 \\
\hline 6 & iobenguane & -0.44911 & 0.036 & $\begin{array}{l}\text { mepenzolate } \\
\text { bromide }\end{array}$ & -0.23048 & 0.038 & $\begin{array}{l}\text { 4- } \\
\text { hydroxyphenazone }\end{array}$ & -0.27839 & 0.004 \\
\hline 7 & sulfadoxine & -0.445275 & 0.038 & $\begin{array}{l}\text { Bephenium } \\
\text { hydroxyl } \\
\text { naphthoate }\end{array}$ & -0.230135 & 0.044 & tocainide & -0.276135 & 0.003 \\
\hline
\end{tabular}




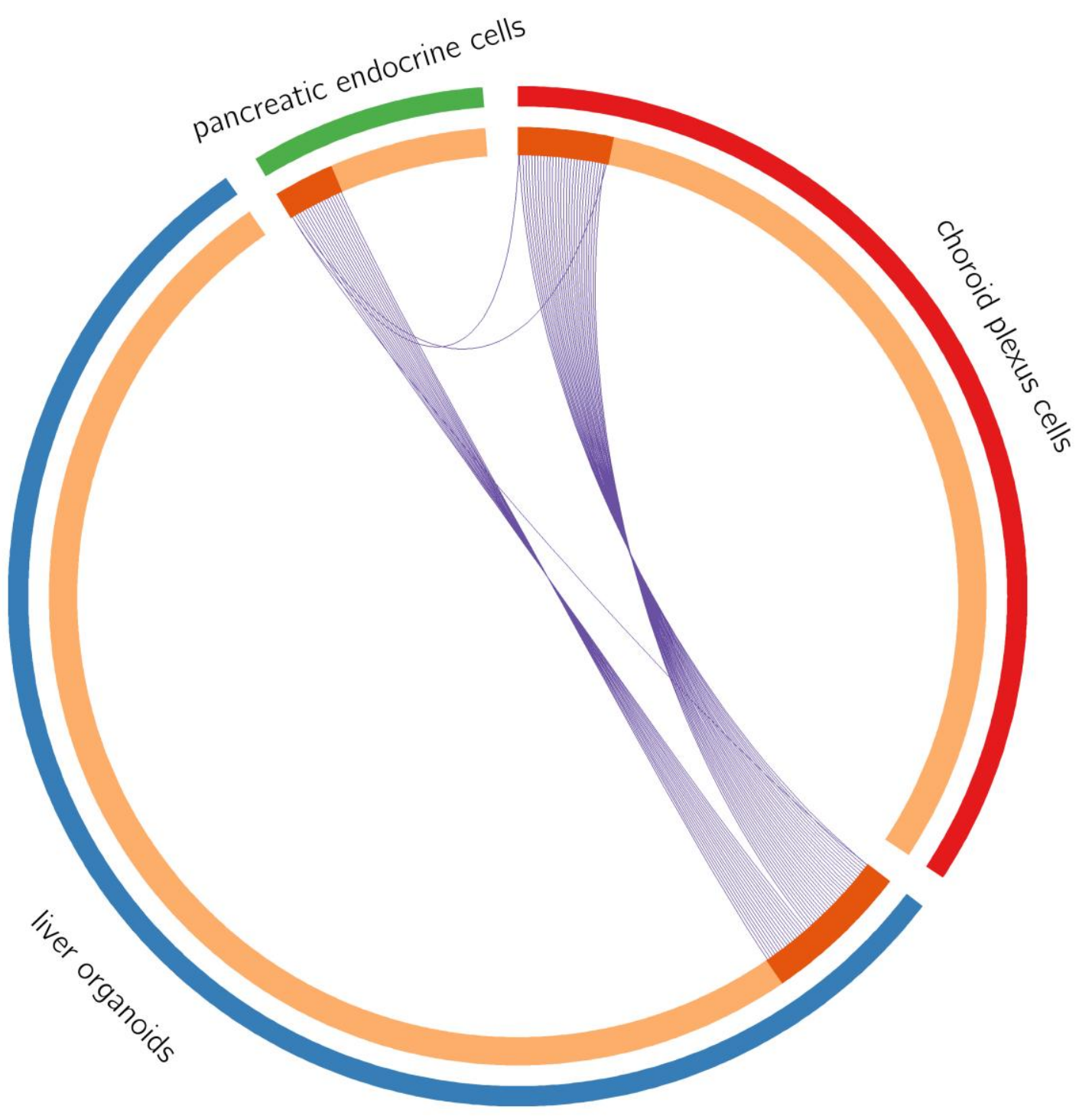

Figure 1. Overlaps between up-regulated genes of SARS-CoV-2 infected hPSCs-derived liver organoids, hPSCs-derived pancreatic endocrine cells, and hiPSCs-derived choroid plexus organoids $24 \mathrm{hpi}$ at the gene level. The inner circle represents the gene lists. Genes unique to a list are shown in light orange and genes that hit multiple lists are colored in dark orange. 


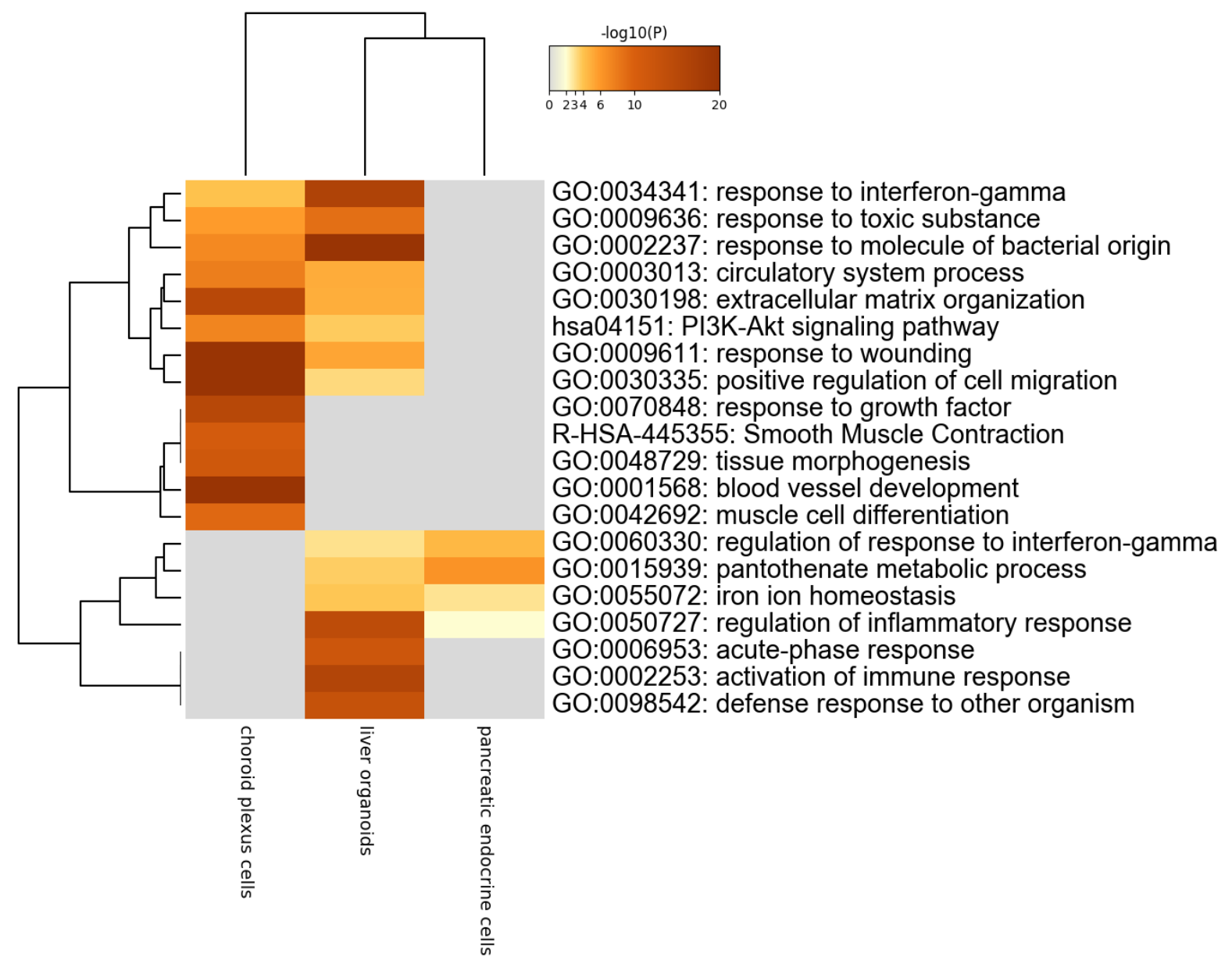

Figure 2. Heatmap of the top-level enriched biological terms across up-regulated genes of SARS-CoV-2 infected hPSCs-derived liver organoids, hPSCs-derived pancreatic endocrine cells, and hiPSCs-derived choroid plexus organoids 24 hpi, colored by p-values. "Log10(P)" is the p-value in log base 10 . 


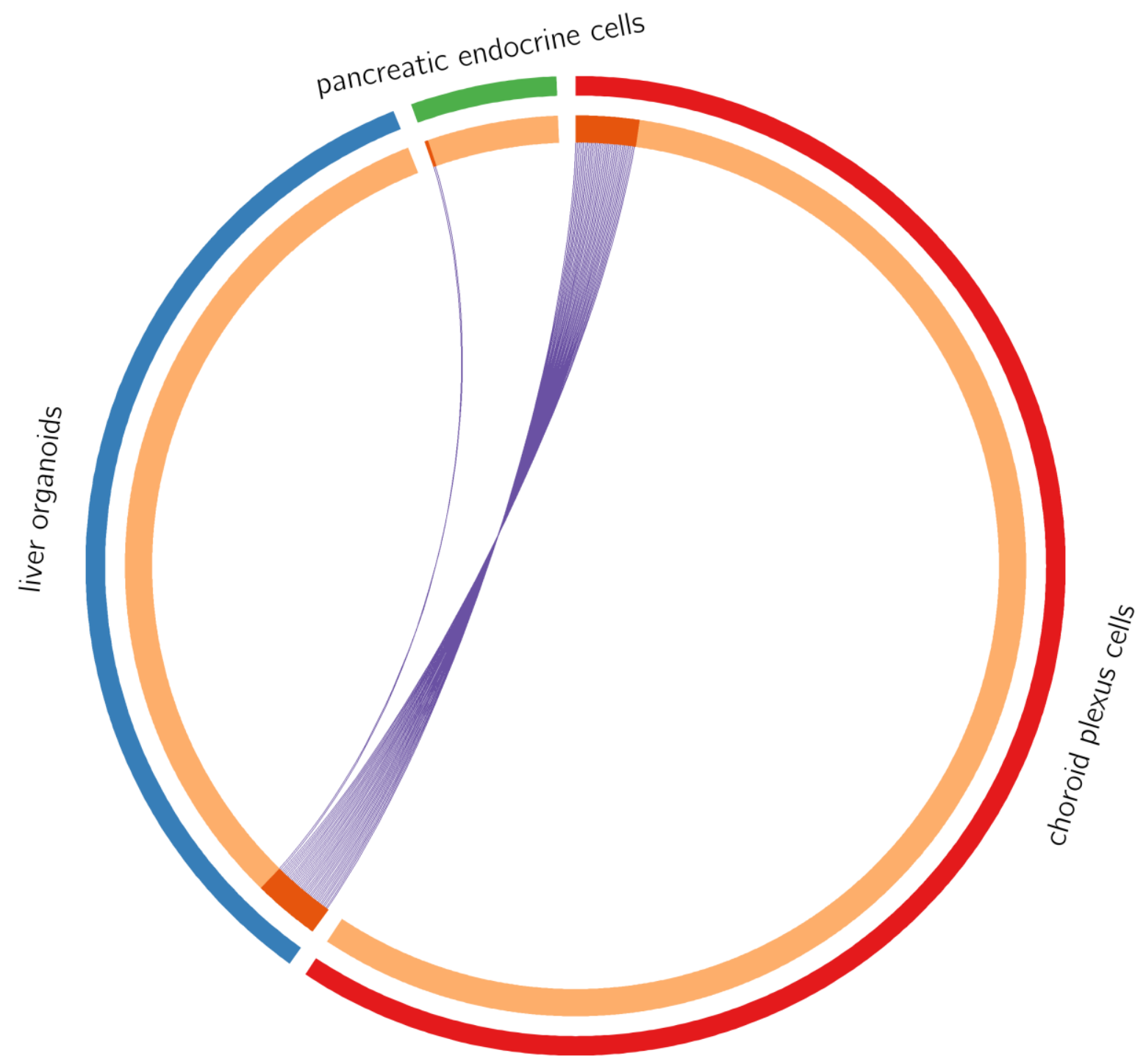

Figure 3. Overlaps between down-regulated genes of SARS-CoV-2 infected hPSCs-derived liver organoids, hPSCs-derived pancreatic endocrine cells, and hiPSCs-derived choroid plexus organoids $24 \mathrm{hpi}$ at the gene level. The inner circle represents the gene lists. Genes unique to a list are shown in light orange and genes that hit multiple lists are colored in dark orange. 


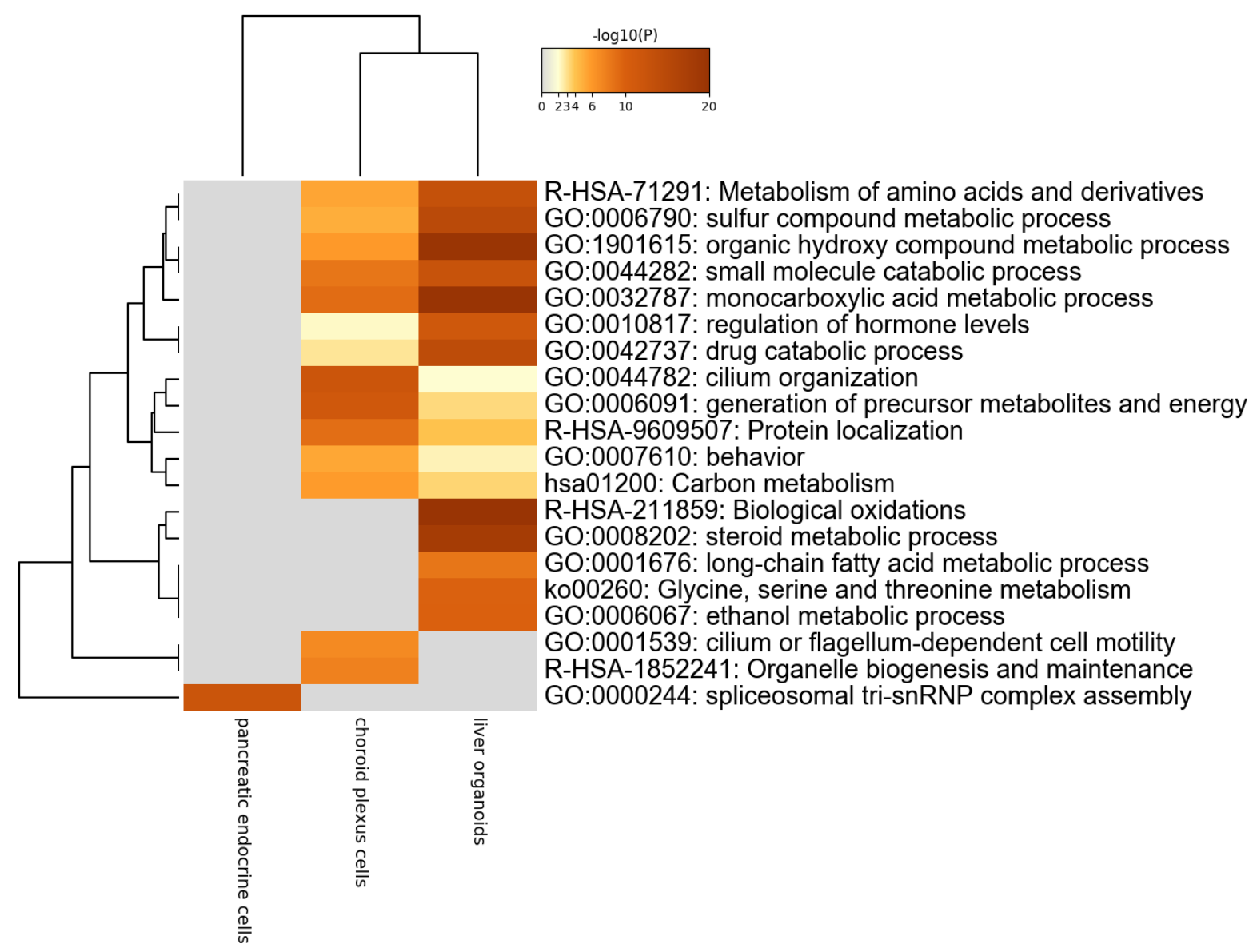

Figure 4. Heatmap of the top-level enriched biological terms across down-regulated genes of SARS-CoV-2 infected hPSCs-derived liver organoids, hPSCs-derived pancreatic endocrine cells, and hiPSCs-derived choroid plexus organoids 24 hpi, colored by p-values. "Log10(P)" is the p-value in log base 10 . 